\title{
Intensity dependence of strong-field double-ionization mechanisms: From field-assisted recollision ionization to recollision-assisted field ionization
}

\author{
A. Emmanouilidou ${ }^{1,2}$ and A. Staudte ${ }^{3}$ \\ ${ }^{1}$ Department of Physics and Astronomy, University College London, Gower Street, London WC1E 6BT, United Kingdom \\ ${ }^{2}$ Chemistry Department, University of Massachusetts at Amherst, Amherst, Massachusetts 01003, USA \\ ${ }^{3}$ Steacie Institute for Molecular Sciences, National Research Council of Canada, 100 Sussex Drive, Ottawa, Ontario, Canada K1A OR6
}

(Received 29 May 2009; published 13 November 2009)

\begin{abstract}
Using a three-dimensional quasiclassical technique we explore molecular double ionization by a linearly polarized, infrared $(800 \mathrm{~nm})$, and ultrashort (6 fs) laser pulse. We first focus on intensities corresponding to the tunneling regime and identify the main ionization mechanisms in this regime. We devise a selection of observables, such as the correlated momenta and the sum of the momenta parallel to the laser field as a function of the interelectronic angle of escape, where all the main mechanisms have distinct traces. Second, we address intensities above but close to the over-the-barrier intensity regime. We find a surprising anticorrelation of electron momenta similar to the experimental observations reported in Phys. Rev. Lett. 101, 053001 (2008) There, however, the anticorrelation was observed in very low intensities corresponding to the multiphoton regime. We discuss the mechanism responsible for the antiparallel two-electron escape.
\end{abstract}

DOI: 10.1103/PhysRevA.80.053415

PACS number(s): $33.80 . \mathrm{Rv}, 34.80 . \mathrm{Gs}, 42.50 . \mathrm{Hz}$

\section{INTRODUCTION}

In the past two decades electron correlation has been established as the underlying mechanism for many important phenomena arising from the interaction of strong laser pulses with matter. One of these phenomena is the dramatically enhanced multiple-ionization yield of atoms (e.g., [1]) and molecules (e.g., [2]) in intense laser pulses. Also called nonsequential multiple ionization, it is the laser driven recollision $[3,4]$ of a field ionized electron with its parent ion that governs the multiple-ionization process up to a certain threshold intensity [5]. Beyond the threshold intensity the contribution of recollision to multiple ionization vanishes and is replaced by a series of independent sequential field ionizations. Using coincidence imaging techniques such as cold-target recoil ion momentum spectroscopy many experiments have succeeded in obtaining highly differential kinematical details of electron correlation in the nonsequential intensity regime (e.g., [6-12]). However, for higher intensities the available experimental data is much less abundant. Correspondingly, the majority of theoretical work has concentrated on the nonsequential intensity regime (e.g., [13-15]).

Here, we report a classical study of electron correlation in $\mathrm{N}_{2}$ molecules subjected to strong laser pulses at intensities well within the nonsequential double-ionization regime (as indicated in Fig. 1), as well as for intensities corresponding to the transition from pure tunneling to over-the-barrier ionization.

First, we consider the intensity regime below the classical over-the-barrier intensity. That is, one electron tunnels in the field-lowered Coulomb potential, then accelerates in the laser field, and finally returns to the core to transfer energy to the remaining electron. In this intensity regime we find that the time of minimum electron approach is close to a zero of the field in agreement with the recollision model [3]. We differentiate the main energy-transfer mechanisms in the recollision of the free electron with the parent ion using the time delay between recollision and ionization of the second elec- tron. Furthermore, we devise a set of asymptotic observables where these mechanisms have distinct traces, such as the sum of the momenta parallel to the laser field as a function of the interelectronic angle of escape.

Second, we consider intensities above, but close to, the over-the-barrier intensity threshold. This is an intermediate regime where a transition takes place from correlated, or nonsequential, to independent, or sequential double ionization. We find that the instant of recollision shifts to a maximum of the field in contrast to the common picture of nonsequential double ionization (NSDI), where the time of recollision is close to a zero of the field. Furthermore, we find that the two electrons asymptotically depart in opposite directions. This is quite unexpected since for very high laser intensities one expects that both electrons ionize almost immediately parallel to the laser field and thus parallel to each other. We discuss how the escape of both electrons in opposite directions can be partly attributed to the laser field sig-

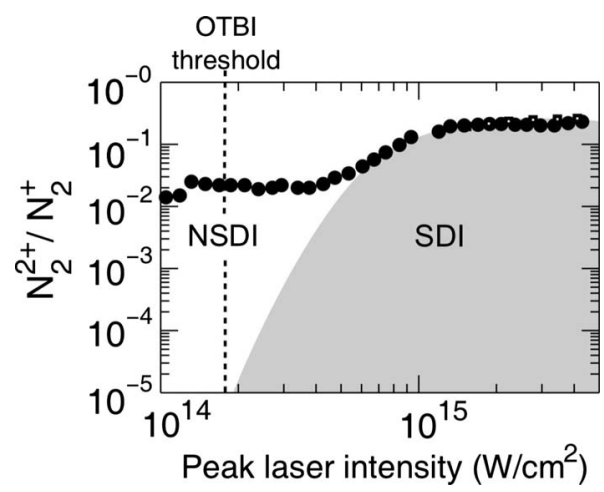

FIG. 1. Ratio of $\mathrm{N}_{2}$ double- and single-ionization yields as a function of laser intensity. Data points were experimentally determined in [16]. At lower intensities nonsequential double ionization (NSDI) is the governing DI mechanism, though at higher intensities sequential double ionization (SDI) eventually dominates. The threshold for over-the-barrier ionization (OTBI) of $\mathrm{N}_{2}$ is about $2 \times 10^{14} \mathrm{~W} / \mathrm{cm}^{2}$. 
nificantly interacting with both electrons before the recollision-in contrast to smaller intensities where the effect of the field on the remaining electron can be neglected; another important factor is that the remaining electron is still bound and thus the effect of the Coulomb potential cannot be neglected.

Addressing the double ionization of strongly driven systems with first-principles quantum-mechanical calculations in all three spatial dimensions is an immense task. Currently, three-dimensional (3D) ab initio quantum-mechanical calculations are only available for the driven He atom [17]. To cope with the highly complex task of tackling the double ionization of diatomic molecules, many studies use numerical quantum approaches of reduced dimensionality (e.g., [18]). Others use judiciously chosen quantum models of reduced dimensionality (e.g., [19]), while some use semianalytical quantum approaches in the framework of the so-called strong-field approximation (SFA) (e.g., [20]), not fully accounting for the Coulomb singularity.

We use a 3D quasiclassical technique that we developed for conservative systems, i.e., the single-photon multiple ionization of atomic systems [21-23]. Recently, we extended this technique to nonconservative systems to treat the correlated electron dynamics of the driven He atom [24]. Here, we further build on this work by tackling more than one atomic centers. Our method is numerically very efficient and treats the Coulomb singularity with no approximation, in contrast to techniques that use "soft-core" potentials. Our method is also important for describing accurately effects such as the striking so-called "fingerlike" structure, which was recently observed in He $[10,11,24,25]$. This structure was attributed to the strong interaction of the rescattering electron with the core-backscattering. In addition, accounting for the Coulomb singularity will be very important in pump-probe setups where vuv or xuv pulses are used. For these highfrequency pulses, the excursion parameter of the electronic motion is smaller than the atomic dimensions, making it very important to accurately incorporate the Coulomb potential.

\section{METHOD}

Our 3D quasiclassical model entails the following steps: We first setup the initial phase-space distribution of the two "active" electrons in the $\mathrm{N}_{2}$ diatomic molecule. Here, we consider only parallel alignment between the molecular axis and the laser electric field. At intensities in the tunneling regime we assume that one electron tunnels through the field-lowered Coulomb potential. For the tunneling rate one can use quantum-mechanical or semiclassical formulas for diatomic molecules (see, e.g., [26-29]). We use the rate provided in Ref. [29]. The longitudinal momentum is zero while the transverse one is provided by a Gaussian distribution [30]. This description is valid as long as the potential barrier is not completely suppressed by the instantaneous laser field $E(t)=E_{0}(t) \cos (\omega t)$. We consider the usual laser wavelength of $800 \mathrm{~nm}$, corresponding to $\omega=0.057$ a.u. (a.u.: atomic units). In our simulation the pulse envelope $E_{0}(t)$ is defined as $E_{0}(t)=E_{0}$ for $0<t<T$ and $E_{0}(t)=E_{0} \cos ^{2}[\omega(t-T) / 8]$ for $T<t<3 T$ with $T$ the period of the field. The threshold for over-the-barrier ionization in neutral $N_{2}$ with an ionization energy of $I_{p 1}=0.5728$ a.u. is reached at a field strength of $E=0.075$ a.u. (corresponding to roughly $2 \times 10^{14} \mathrm{~W} / \mathrm{cm}^{2}$ ).

Above $2 \times 10^{14} \mathrm{~W} / \mathrm{cm}^{2}$ the laser field allows an unhindered electron escape and therefore the initial phase space is modeled by a double electron microcanonical distribution [31]. However, it is important to note that in setting up the initial phase-space distribution we transition smoothly from the tunneling to the over-the-barrier intensity regime. Namely, we assign a random number to the phase $\phi$ of the laser field when the first electron is ionized, see [25,32]. If the phase $\phi$ corresponds to an instantaneous strength of the laser field $E(\phi)$ that leaves the electron below the barrier, then we use the initial conditions dictated by the tunneling model. If the instantaneous field strength pushes the barrier below the $I_{p 1}$ of that electron, then we use the microcanonical distribution to setup the initial phase-space distribution. This choice of initial conditions has proven successful in past studies [25] in modeling the experimental ratio of double versus single ionization for long laser pulses [16]. With our approach we ensure a smooth transition of the initial phasespace distribution as we change the intensity. Even at an intensity of $3 \times 10^{14} \mathrm{~W} / \mathrm{cm}^{2}$ still about $75 \%$ of the doubleionization probability corresponds to trajectories initialized using the tunneling model, while $25 \%$ of the probability corresponds to trajectories initialized using the microcanonical distribution.

After setting up the initial phase-space distribution we transform to a new system of coordinates, the so-called "regularized" coordinates [33]. This transformation is exact and explicitly eliminates the Coulomb singularity. This step is more challenging for molecular systems since one has to "regularize" with respect to more than one atomic centers versus one atomic center for atoms. We regularize using the global regularization scheme described in Ref. [34]. Finally, we use the classical trajectory Monte Carlo (CTMC) method for the time propagation [35]. The propagation involves the 3D four-body Hamiltonian in the laser field with "frozen" nuclei,

$$
\begin{aligned}
H= & \sum_{i=1}^{2}\left[\frac{p_{i}^{2}}{2}-\frac{1}{\left|\boldsymbol{R} / 2-\boldsymbol{r}_{i}\right|}-\frac{1}{\left|-\boldsymbol{R} / 2-\boldsymbol{r}_{i}\right|}\right]+\frac{1}{\left|\boldsymbol{r}_{1}-\boldsymbol{r}_{2}\right|} \\
& +\left(\boldsymbol{r}_{1}+\boldsymbol{r}_{2}\right) \cdot \boldsymbol{E}(t),
\end{aligned}
$$

where $E(t)$ is the laser electric field polarized along the $z$ direction and further defined as detailed above, and $\boldsymbol{R}$ is the internuclear distance.

\section{RESULTS}

Recollision as a subcycle, true attosecond process permits simulations using few cycle pulses to describe the main signatures of electron correlation that are manifested in multicycle pulse experiments [10]. Details of electron correlation as the branching ratio of the different NSDI pathways do depend on the pulse duration [36]. In few cycle pulses the dominant channel of NSDI is the simultaneous ejection (SE) of both electrons upon impact of the recolliding electron. The simultaneous ejection channel is responsible for the NSDI 

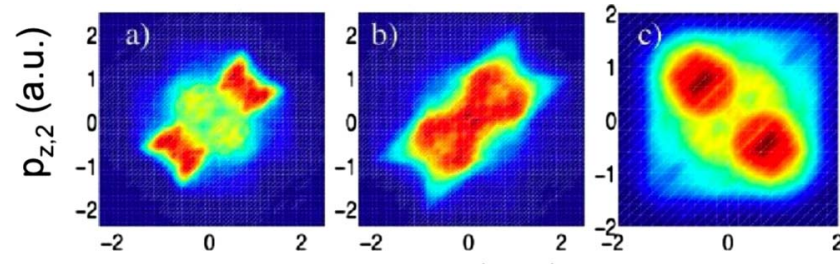

$$
p_{z, 1} \text { (a.u.) }
$$
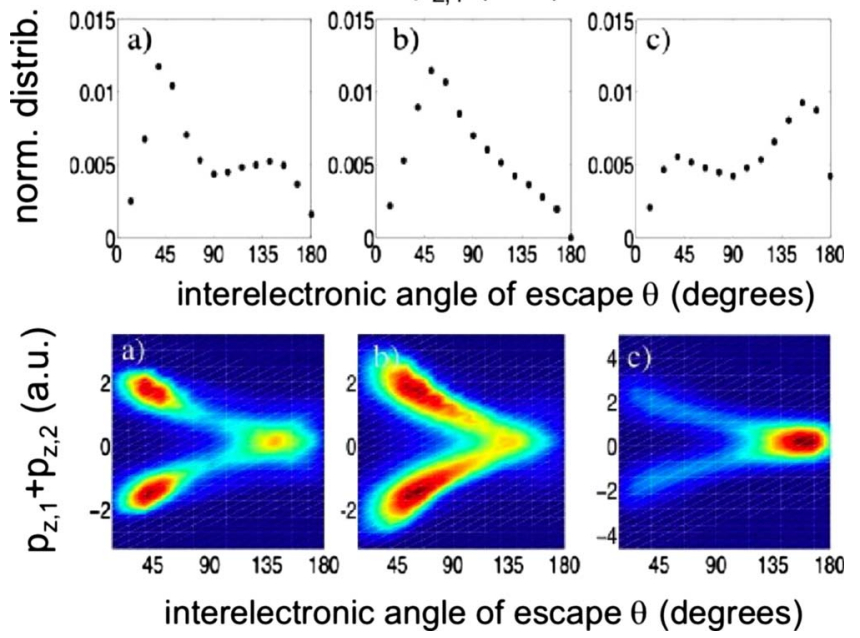

FIG. 2. (Color online) Intensity dependence of electron correlation in the double ionization of $\mathrm{N}_{2}$ in a three-cycle $800 \mathrm{~nm}$ pulse. Columns (a)-(c) correspond to increasing intensities $10^{14} \mathrm{~W} / \mathrm{cm}^{2}$, $1.5 \times 10^{14} \mathrm{~W} / \mathrm{cm}^{2}$, and $3 \times 10^{14} \mathrm{~W} / \mathrm{cm}^{2}$, respectively. Top row: correlated momenta parallel to the polarization axis; Middle row: distribution of the interelectronic angle of escape $\theta$; Bottom row: sum of the parallel momenta vs $\theta$.

hallmark: a double hump structure in the sum of the momenta parallel to the laser field around $4 \sqrt{U_{p}}$. Here, $2 \sqrt{U_{p}}$ is the maximum velocity an electron can acquire from its interaction with the field and with $U_{p}=E_{0}^{2} /\left(4 \omega^{2}\right)$ being the ponderomotive energy.

As the laser intensity is increased from $1 \times 10^{14} \mathrm{~W} / \mathrm{cm}^{2}$ to $3 \times 10^{14} \mathrm{~W} / \mathrm{cm}^{2}$ a transition takes place as evidenced by the correlated momenta of the two escaping electrons shown in Figs. 2(a)-2(c). At $10^{14} \mathrm{~W} / \mathrm{cm}^{2}$ both electrons escape with very similar momenta [Fig. 2(a)]. Already at 1.5 $\times 10^{14} \mathrm{~W} / \mathrm{cm}^{2}$ the distribution changes significantly and develops an X-like shape [Fig. 2(b)]. However, the interelectronic angle of escape $\theta$ (the angle between the asymptotic electron momenta) is generally less than $90^{\circ}$. In contrast, at $3 \times 10^{14} \mathrm{~W} / \mathrm{cm}^{2}$ the interelectronic angle of escape becomes larger than $90^{\circ}$. In fact, the two electrons tend to escape with opposite momenta [Fig. 2(c)]. This transition is also evident when one considers the distribution of $\theta$ [Fig. 2(c), medium panel] with a maximum around $165^{\circ}$. We note that our model does not account for depletion of the initial state in the overthe-barrier regime. For that reason we only present the correlated momenta for intermediate intensities up to 3 $\times 10^{14} \mathrm{~W} / \mathrm{cm}^{2}$ where the tunneling process still dominates as 3:1, thereby rendering the effect of depletion small. Finally, we note parenthetically that at $3 \times 10^{14} \mathrm{~W} / \mathrm{cm}^{2}$ overthe-barrier ionization of the first electron becomes possible, whereas NSDI is still the dominating mechanism in the liberation of the second electron (compare Fig. 1).

\section{A. Tunneling regime}

We now proceed to identify the double-ionization pathways for intensities in the tunneling regime. Two main mechanisms for nonsequential double ionization [19] are well established: SE upon rescattering and recollisioninduced excitation with subsequent field ionization (RESI).

In the SE mechanism both electrons are simultaneously ejected with small but equal momenta, with the result that the asymptotic momentum components along the polarization axis are mainly dictated by the vector potential of the laser field at the time of ejection. This agrees with Fig. 2(a) where the maximum of the interelectronic angle of escape is around $30^{\circ}$. In RESI the rescattering electron only excites the remaining electron, which is ionized at a subsequent maximum of the laser field [37-40].

In Figs. 3 and 4 we can clearly identify SE and RESI but also two other mechanisms. Namely, in Fig. 3c) we identify a NSDI pathway which starts out with excitation upon recollision, but in contrast to RESI the excited electron ionizes around a subsequent zero of the field. We label this mechanism NSE2. In Fig. 3(d) we identify yet another mechanism that involves the formation of a doubly-excited complex at the recollision with both electrons ionizing at a later time as discussed in $[19,36]$ — we refer to this mechanism as the double excitation (DE) pathway. Previously, it was shown how the double-ionization mechanisms have distinct traces in the spatial electron density [19]. In the current work, we find that those different mechanisms also leave distinct traces in the observable momentum space.

When electrons ionize through the SE pathway, they escape with large momenta and with interelectronic angles less than $90^{\circ}$; in ionization through the RESI pathway the sum of the parallel components of the momenta is smaller and the electrons typically escape with angles larger than those for the SE mechanism. When the electrons escape through the NSE2 or the DE pathway the sum of the momenta is zero with large interelectronic angles of escape. However, the two pathways have different correlated momenta as shown in Figs. 3 and 4 (medium panel) - larger electron momenta are characteristic for the NSE2 mechanism. The SE and NSE2 are the dominant mechanisms for a three-cycle laser pulse accounting for roughly $44 \%$ and $39 \%$, respectively, for a laser intensity of $10^{14} \mathrm{~W} / \mathrm{cm}^{2}$ and for $54 \%$ and $28 \%$, respectively, for a laser intensity of $1.5 \times 10^{14} \mathrm{~W} / \mathrm{cm}^{2}$, while RESI is around $10 \%$ in both cases. The contribution of the RESI and DE mechanisms increases significantly with increasing laser-pulse durations.

We note that the identification of the different mechanisms is based on the fact that the recolliding electron returns to the core, i.e., on the existence of an instant of closest approach to the remaining electron. We further verify the distinction between the different mechanisms by analyzing the time of ionization after recollision shown in the bottom panels of Figs. 3 and 4. To determine the time of ionization we make use of the "compensated energy" [41] for each electron. The compensated energy is the electron's potential energy with respect to the two nuclei plus its kinetic energy resulting from the canonical momentum due to the presence 

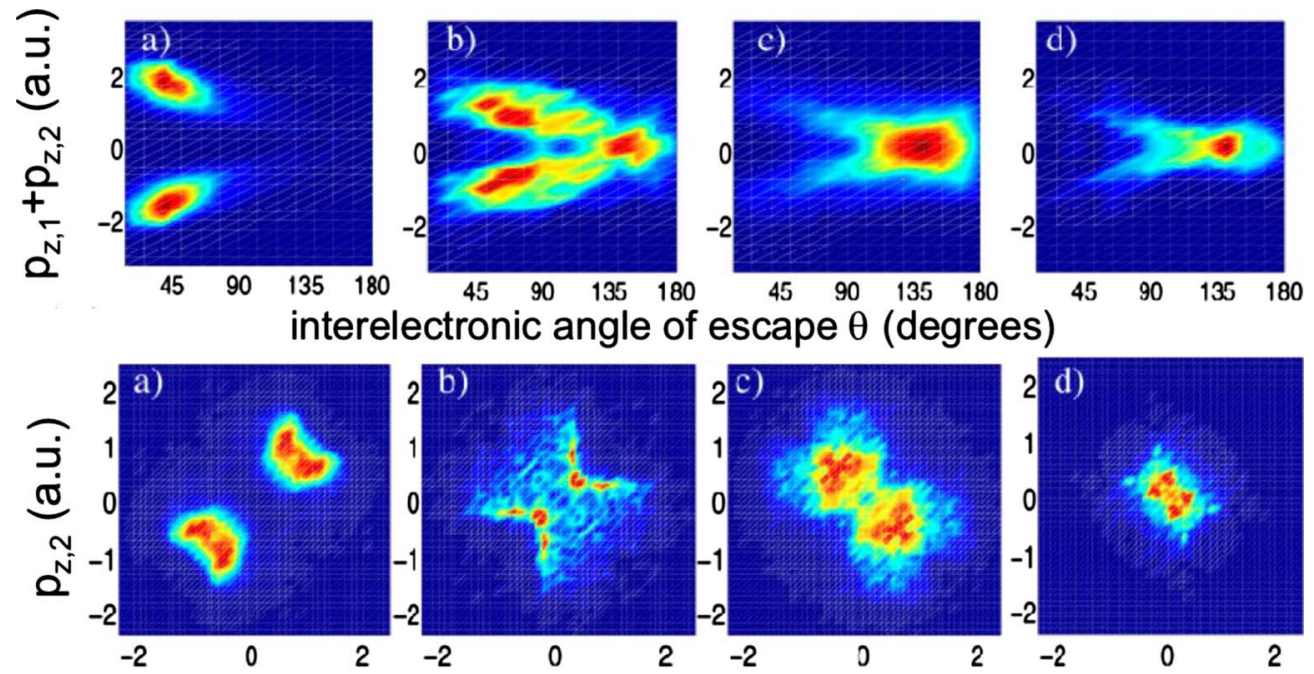

$p_{z, 1}$ (a.u.)

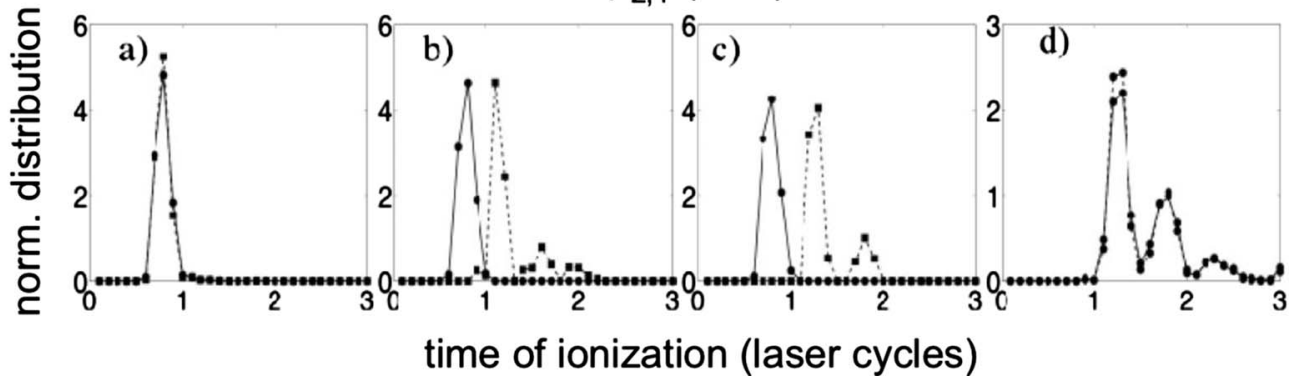

FIG. 3. (Color online) For $10^{14} \mathrm{~W} / \mathrm{cm}^{2}$ we identify four different double-ionization mechanisms depending on each electron's time of ionization: the different columns refer to (a) the SE, (b) the RESI, (c) the NSE2, and (d) the DE mechanisms. Top row: the sum of the momenta of the two electrons as a function of the interelectronic angle of escape; Middle row: the correlated momenta of the two electrons; Bottom row: the ionization time of each electron in units of laser cycles.

of the field. Here we define ionization as the instant when the compensated energy becomes positive and remains positive for all subsequent times. While being an approximate way to define the ionization time for each electron it proves useful for the identification of the different mechanisms.

\section{B. Over-the-barrier regime}

Now we are turning toward the results obtained at an intermediate intensity regime which is high enough that over-the-barrier ionization of the first electron becomes possible but low enough that the second electron cannot ionize without the energy transfer in a recollision with the first electron [see Fig. 2(c)]. The most striking feature of this intensity regime is the strong anticorrelation between the electron momenta, which resembles experimental observations by Liu et al. [12]. There, however, the anticorrelation was observed at intensities well below the classical threshold for recollision double ionization. Since at these intensities a single recollision does not provide sufficient energy to release a second electron a repeated energy transfer from the continuum electron via multiple field-assisted recollisions was held responsible for this effect.

However, in the intermediate intensity regime currently under consideration the energy of the recolliding electron easily exceeds the binding energy of the second electron. At
$3 \times 10^{14} \mathrm{~W} / \mathrm{cm}^{2}$ the impact energy at recollision can be as high as $54 \mathrm{eV}$ while the ionization energy of $\mathrm{N}_{2}^{+}$is approximately $I_{p 2}=30 \mathrm{eV}$. While this permits ionization also through shorter trajectories, as we discuss below, experiments have shown [10] that when excess energy is available only the minimum energy necessary for ionization is transferred in the collision and the recolliding electron retains most of the excess energy.

To identify whether these "soft" collisions are indeed responsible for the observed anticorrelation we examine in Fig. 5 the temporal evolution of the kinetic and potential energy of both electrons for laser intensities $10^{14} \mathrm{~W} / \mathrm{cm}^{2}$ and $3 \times 10^{14} \mathrm{~W} / \mathrm{cm}^{2}$. The amplitude in the potential-energy changes reveals that the collision for $3 \times 10^{14} \mathrm{~W} / \mathrm{cm}^{2}$ (thin lines) takes place further out from the nucleus if compared to the collision at $10^{14} \mathrm{~W} / \mathrm{cm}^{2}$ (thick lines). This interpretation is further corroborated by the presence of the fingerlike structure in the correlated momenta in Fig. 2(a) for $10^{14} \mathrm{~W} / \mathrm{cm}^{2}$ and its absence in Fig. 2(c) for 3 $\times 10^{14} \mathrm{~W} / \mathrm{cm}^{2}$. The fingerlike structure was attributed in former studies of the strongly driven He atom to strong backscattering from the nucleus $[10,24,25]$. Finally, in Fig. 5 the temporal evolution of the kinetic energy shows that there is a smaller relative change in the kinetic energy of the two electrons for $3 \times 10^{14} \mathrm{~W} / \mathrm{cm}^{2}$ around $T / 2$ when compared to the kinetic-energy change for $10^{14} \mathrm{~W} / \mathrm{cm}^{2}$ around $3 / 4 T$. 

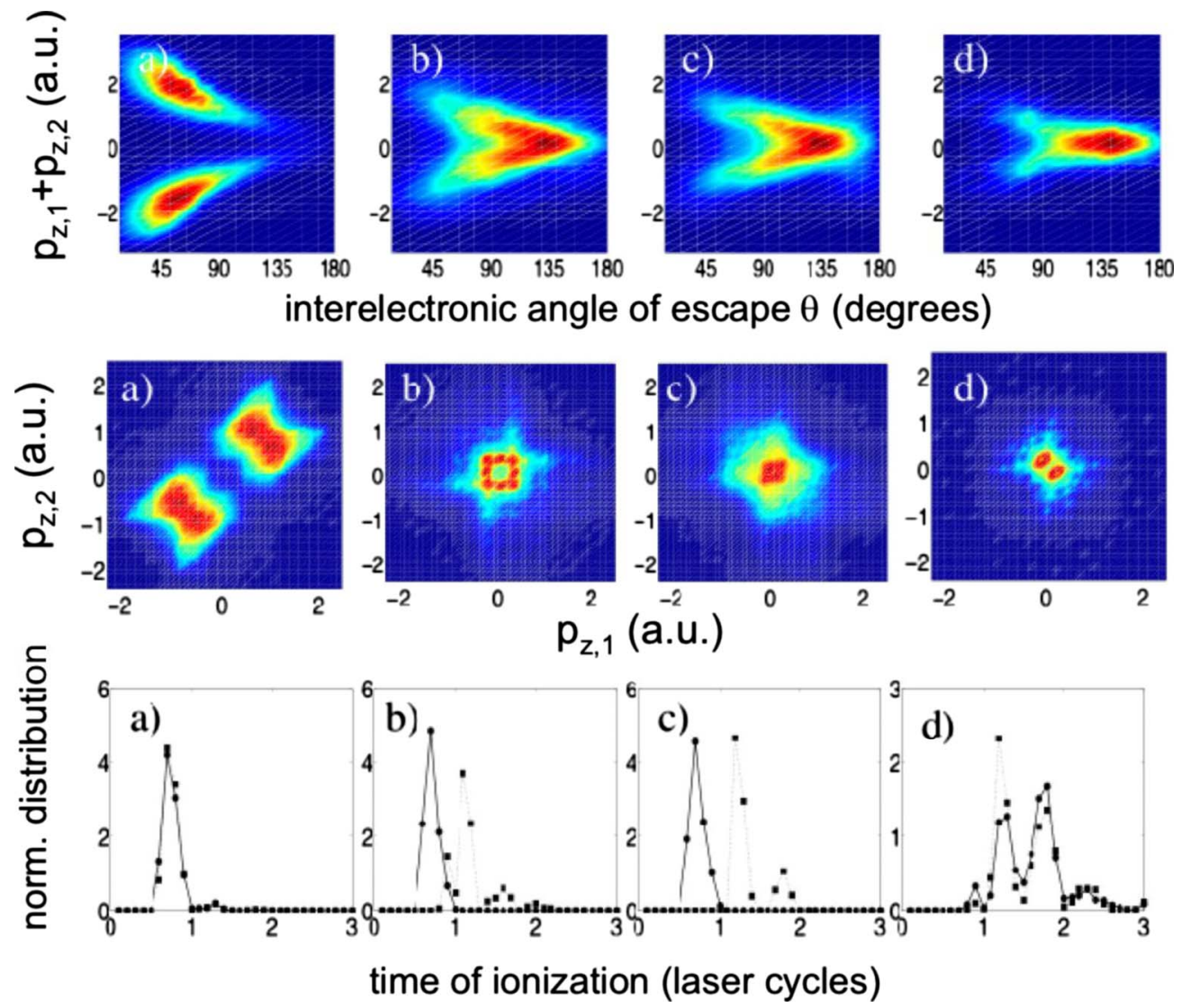

FIG. 4. (Color online) Same as for Fig. 3 but for $1.5 \times 10^{14} \mathrm{~W} / \mathrm{cm}^{2}$.

At $3 \times 10^{14} \mathrm{~W} / \mathrm{cm}^{2}$ soft collisions with large impact parameter seem to be favored due to the prevalence of short trajectories in the total double-ionization yield. In Fig. 6 we identify the short trajectories by analyzing the temporal structure of the recollision process. We plot the phase of the field when the first electron is "launched" as a function of the instant of double ionization (according to our previous definition of ionization time using the compensated energy). In the tunneling regime the recolliding electron is launched shortly after the maximum of the laser field [Fig. 6(a) and $6(b)]$, i.e., the phase $\phi$ of the laser field is close to zero (we use a cosine field). The majority of double-ionization trajec-
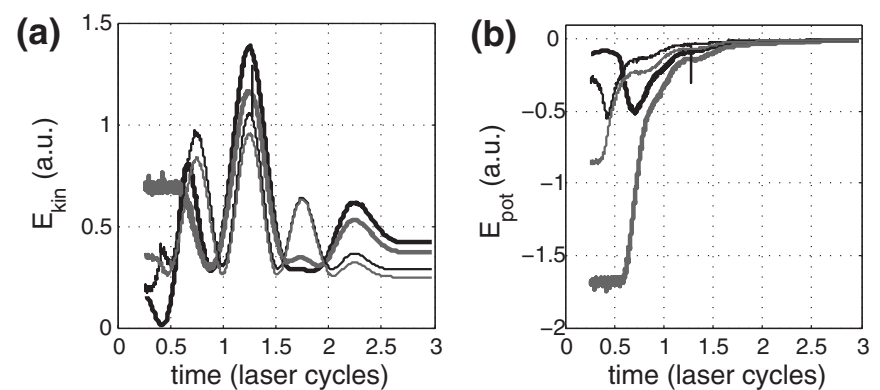

FIG. 5. Time dependence of kinetic energy (left panel) and potential energy (right panel) for the recolliding electron (black lines) and the initially bound electron (gray lines). Shown is the average over all double-ionization trajectories at $10^{14} \mathrm{~W} / \mathrm{cm}^{2}$ (thick lines) and $3 \times 10^{14} \mathrm{~W} / \mathrm{cm}^{2}$ (thin lines). tories then recollide at around 3/4 into the laser cycle (zero of the laser field) corresponding to long trajectories. In contrast, in the over-the-barrier intensity regime the recolliding electron is launched when the phase of the laser field is larger, see Fig. 6(c), corresponding to short trajectories. We find that the time delay between "launching" of the first electron (recolliding electron) and the first recollision, i.e., the maximum of the electron-electron interaction energy, decreases from $2 / 3 T$ at $1 \times 10^{14} \mathrm{~W} / \mathrm{cm}^{2}$ to $1 / 2 T$ at $3 \times 10^{14} \mathrm{~W} / \mathrm{cm}^{2}$. Although short trajectories have less than the maximum impact energy of $3.17 U_{p}$ they can be energetic enough at high intensities to release the second electron. However, the electron energy transfer becomes temporally less localized when the intensity is increased to $3 \times 10^{14} \mathrm{~W} / \mathrm{cm}^{2}$.

From the above it is clear that a transfer of energy through the recollision event is still necessary for the bound electron to ionize for intensities in the intermediate regime. However, the laser field for the higher intensities influences the motion of the bound electron even before the recollision process. In contrast for the smaller intensities the asymptotic momenta components along the polarization axis are roughly dictated by the vector potential at the time of recollision (around $2 / 3 T$ ). The significant role for higher intensities of the laser field even before the recollision process can be also inferred from Fig. 7. Namely, depending on the time delay between the time of launching of the first electron and the time of double ionization, the two electrons escape either parallel or opposite to each other. In particular, the prevailing interelec- 


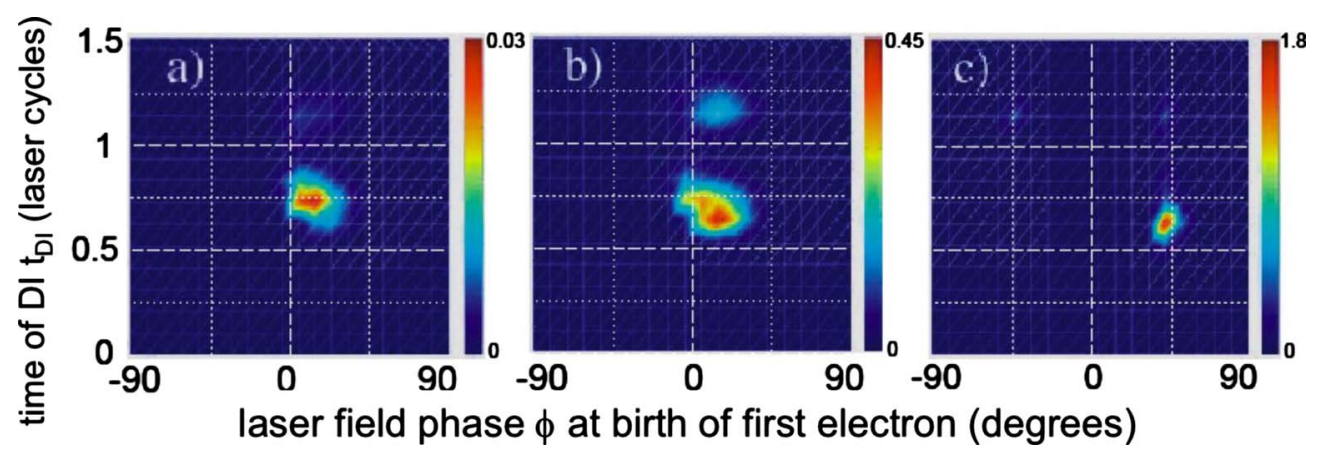

FIG. 6. (Color online) Instant of double ionization $t_{D I}$ as a function of the phase $\phi$ of the laser field when the first electron is "launched." The laser intensity is (a) $10^{14} \mathrm{~W} / \mathrm{cm}^{2}$, (b) $1.5 \times 10^{14} \mathrm{~W} / \mathrm{cm}^{2}$, and (c) $3 \times 10^{14} \mathrm{~W} / \mathrm{cm}^{2}$.

tronic angle larger than $90^{\circ}$ is due to the fact that the second electron ionizes approximately $\mathrm{T} / 2$ after the first electron is launched. With the vector potential changing sign within half a period, this results in electrons escaping in opposite directions.

We will now discuss the limitations of our model with respect to the high intensity results. Our model does not incorporate sequential double ionization, i.e., tunneling of the second electron independent of the launching of the first electron. The independent tunneling of both electrons is known to yield uncorrelated electron momenta [6], which would obscure the observed anticorrelation. However, at 3 $\times 10^{14} \mathrm{~W} / \mathrm{cm}^{2}$ sequential double ionization can be neglected (compare Fig. 1).

Further, our simulation does not include spatial intensity averaging as it is virtually unavoidable in the experiment. Contribution of lower peak intensities to the overall DI signal would partially mask the anticorrelation. This effect is expected to become increasingly important for higher intensities.

Finally, one may legitimately ask to what extend the above finding is a molecular effect. In a recent study of the double ionization of the strongly driven He atom [24] the correlated momenta did not exhibit a pattern of electrons ejected in opposite directions. One possible explanation for the difference we find between $\mathrm{He}$ and $\mathrm{N}_{2}$ is that while for $\mathrm{N}_{2}$, there is a significant range of intensities where the overthe-barrier regime and the nonsequential one overlap-the overlap characterizes the intermediate regime discussed in

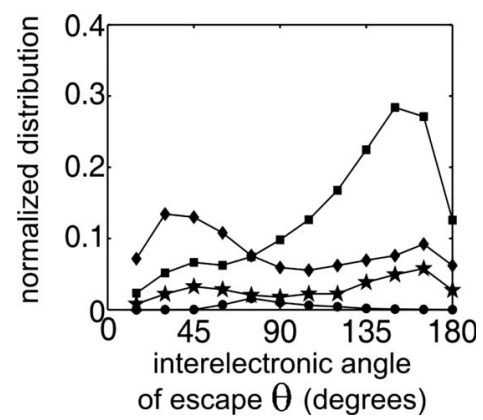

FIG. 7. For $3 \times 10^{14} \mathrm{~W} / \mathrm{cm}^{2}$ we show the distribution of the interelectronic angle when the double-ionization time is from $T / 4$ to $T / 2$ (circles); from $T / 2$ to $3 / 4 T$ (squares); from $3 / 4 T$ to $T$ (stars); and from $T$ to $5 / 4 T$ (diamonds). the current paper-this is not the case for He. Namely, for $\mathrm{He}$ at the intensities where the over-the-barrier regime is reached sequential ionization dominates over the nonsequential one. However, since our explanation of the anticorrelation does not necessitate molecular structure we would expect in principle a similar effect in atoms. Future studies in other atomic and molecular species can explore further whether the anticorrelation effect is generally observed for intermediate intensities where there is an overlap of the over-the-barrier intensity regime and the regime where nonsequential ionization dominates over the sequential ionization.

\section{CONCLUSION}

In conclusion, we have studied the nonsequential double ionization up to intensities where our classical model permits an intuitive definition of recollision. In the tunneling regime we identify two new double-ionization mechanisms and devise asymptotic observables with distinct traces of these ionization mechanisms. For intensities corresponding to the over-the-barrier ionization but still within the nonsequential double-ionization regime, we surprisingly find that both electrons are ejected with opposite momenta. In this intermediate intensity regime it is the field interaction with the second bound electron that permits soft recollisions to assist the second ionization step. The combination of soft recollision and timing between the two ionization steps forms the underlying mechanism for the observed anticorrelation of the electron momenta. An anticorrelation pattern was also observed in [12] for small intensities where the mechanism responsible was identified as multiple inelastic field-assisted recollisions. These results motivate a revision of the common notion that nonsequential double ionization necessarily means emission of both electrons with identical momenta.

\section{ACKNOWLEDGMENTS}

The authors gratefully acknowledge discussions with Paul Corkum, André Bandrauk, and Carla Figueira de Morison Faria. A.E. gratefully acknowledges funding from EPSRC under Grant No. EPSRC/H0031771, as well as from NSF, Grant No. NSF/0855403. 
[1] B. Walker, B. Sheehy, L. F. DiMauro, P. Agostini, K. J. Schafer, and K. C. Kulander, Phys. Rev. Lett. 73, 1227 (1994).

[2] A. S. Alnaser, T. Osipov, E. P. Benis, A. Wech, B. Shan, C. L. Cocke, X. M. Tong, and C. D. Lin, Phys. Rev. Lett. 91, 163002 (2003).

[3] P. B. Corkum, Phys. Rev. Lett. 71, 1994 (1993).

[4] K. J. Schafer, B. Yang, L. F. DiMauro, and K. C. Kulander, Phys. Rev. Lett. 70, 1599 (1993).

[5] A. Rudenko, Th. Ergler, K. Zrost, B. Feuerstein, V. L. B. de Jesus, C. D. Schröter, R. Moshammer, and J. Ullrich, J. Phys. B 41, 081006 (2008).

[6] Th. Weber, H. Giessen, M. Weckenbrock, G. Urbasch, A. Staudte, L. Spielberger, O. Jagutzki, V. Mergel, M. Vollmer, and R. Dörner, Nature (London) 405, 658 (2000).

[7] E. Eremina, X. Liu, H. Rottke, W. Sandner, A. Dreischuh, F. Lindner, F. Grasbon, G. G. Paulus, H. Walther, R. Moshammer, B. Feuerstein, and J. Ullrich, J. Phys. B 36, 3269 (2003).

[8] V. L. B. de Jesus, A. Rudenko, B. Feuerstein, K. Zrost, C. D. Schröter, R. Moshammer, and J. Ullrich, J. Electron Spectrosc. Relat. Phenom. 141, 127 (2004).

[9] M. Weckenbrock, D. Zeidler, A. Staudte, Th. Weber, M. Schöffler, M. Meckel, S. Kammer, M. Smolarski, O. Jagutzki, V. R. Bhardwaj, D. M. Rayner, D. M. Villeneuve, P. B. Corkum, and R. Dörner, Phys. Rev. Lett. 92, 213002 (2004).

[10] A. Staudte, C. Ruiz, M. Schöffler, S. Schössler, D. Zeidler, Th. Weber, M. Meckel, D. M. Villeneuve, P. B. Corkum, A. Becker, and R. Dörner, Phys. Rev. Lett. 99, 263002 (2007).

[11] A. Rudenko, V. L. B. de Jesus, Th. Ergler, K. Zrost, B. Feuerstein, C. D. Schröter, R. Moshammer, and J. Ullrich, Phys. Rev. Lett. 99, 263003 (2007).

[12] Y. Liu, S. Tschuch, A. Rudenko, M. Dürr, M. Siegel, U. Morgner, R. Moshammer, and J. Ulrich, Phys. Rev. Lett. 101, 053001 (2008).

[13] P. J. Ho, Phys. Rev. A 72, 045401 (2005).

[14] S. L. Haan, L. Breen, A. Karim, and J. H. Eberly, Phys. Rev. Lett. 97, 103008 (2006).

[15] D. Y. Bondar, Phys. Rev. A 79, 023417 (2009).

[16] C. Cornaggia and Ph. Hering, Phys. Rev. A 62, 023403 (2000).

[17] J. S. Parker, B. J. S. Doherty, K. T. Taylor, K. D. Schultz, C. I. Blaga, and L. F. DiMauro, Phys. Rev. Lett. 96, 133001 (2006).

[18] A. D. Bandrauk and H. Z. Lu, Phys. Rev. A 72, 023408 (2005).
[19] S. Baier, A. Becker, and L. Plaja, Phys. Rev. A 78, 013409 (2008).

[20] C. Figueira de Morison Faria, T. Shaaran, X. Liu, and W. Yang, Phys. Rev. A 78, 043407 (2008).

[21] A. Emmanouilidou and J. M. Rost, J. Phys. B 39, 4037 (2006).

[22] A. Emmanouilidou, Phys. Rev. A 75, 042702 (2007).

[23] A. Emmanouilidou and J. M. Rost, Phys. Rev. A 75, 022712 (2007).

[24] A. Emmanouilidou, Phys. Rev. A 78, 023411 (2008).

[25] D. F. Ye, X. Liu, and J. Liu, Phys. Rev. Lett. 101, 233003 (2008).

[26] X. M. Tong, Z. X. Zhao, and C. D. Lin, Phys. Rev. A 66, 033402 (2002).

[27] I. V. Litvinyuk, K. F. Lee, P. W. Dooley, D. M. Rayner, D. M. Villeneuve, and P. B. Corkum, Phys. Rev. Lett. 90, 233003 (2003).

[28] T. K. Kjeldsen and L. B. Madsen, J. Phys. B 37, 2033 (2004).

[29] Y. Li, J. Chen, S. P. Yang, and J. Liu, Phys. Rev. A 76, 023401 (2007).

[30] J. Klein, F. Beil, and T. Halfmann, Phys. Rev. Lett. 99, 113003 (2007)

[31] L. Meng, C. O. Reinhold, and R. E. Olson, Phys. Rev. A 40, 3637 (1989).

[32] T. Brabec, M. Yu. Ivanov, and P. B. Corkum, Phys. Rev. A 54, R2551 (1996).

[33] P. Kustaanheimo and E. Stiefel, J. Reine Angew. Math. 218, 204 (1965).

[34] D. C. Heggie, Celest. Mech. 10, 217 (1974).

[35] R. Abrines, I. C. Percival, and N. A. Valentine, Proc. Phys. Soc. London 89, 515 (1966).

[36] J. S. Prauzner-Bechcicki, K. Sacha, B. Eckhardt, and J. Zakrzewski, Phys. Rev. A 71, 033407 (2005).

[37] K. J. LaGattuta and J. S. Cohen, J. Phys. B 31, 5281 (1998).

[38] R. Kopold, W. Becker, H. Rottke, and W. Sandner, Phys. Rev. Lett. 85, 3781 (2000).

[39] B. Feuerstein, R. Moshammer, D. Fischer, A. Dorn, C. D. Schröter, J. Deipenwisch, J. R. Crespo Lopez-Urrutia, C. Höhr, P. Neumayer, J. Ullrich, H. Rottke, C. Trump, M. Wittman, G. Korn, and W. Sandner, Phys. Rev. Lett. 87, 043003 (2001).

[40] G. L. Yudin and M. Yu. Ivanov, Phys. Rev. A 63, 033404 (2001).

[41] J. G. Leopold and I. C. Percival, J. Phys. B 12, 709 (1979). 